\title{
OPPORTUNITIES OF ENERGY SAVING LIGHTING FOR SUSTAINABLE DEVELOPMENT
}

\author{
Ruzena Kralikova, Frantisek Koblasa, Emil Wessely, \\ Miriam Pinosova \& Laura Dzunova
}
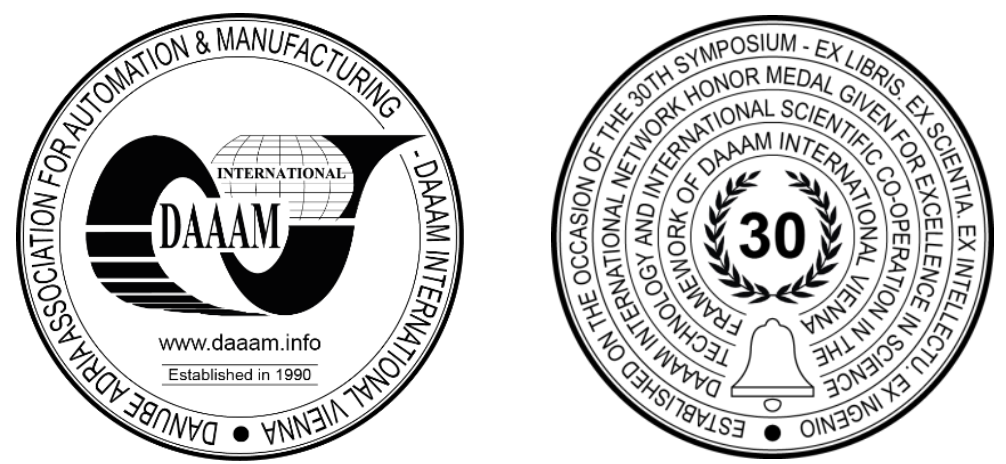

This Publication has to be referred as: Kralikova, R[uzena]; Koblasa, F[rantisek]; Wessely, E[mil]; Pinosova, M[iriam] \& Dzunova, L[aura] (2020). Opportunities of Energy Saving Lighting for Sustainable Development, Proceedings of the 31st DAAAM International Symposium, pp.0044-0048, B. Katalinic (Ed.), Published by DAAAM International, ISBN 978-3-902734-29-7, ISSN 1726-9679, Vienna, Austria

DOI: $10.2507 / 31$ st.daaam.proceedings.006

\begin{abstract}
Human factors and lighting are concerned with the relations between the user, equipment and their environments. It considers the user's capabilities and limitation and seeks to ensure that tasks, functions, information and the environment suits each user. Current lighting solutions present crucial challenges for both local and global sustainable development. Light is becoming increasingly important in the industrial sector. The industrial lighting can cut operating costs and improve visual conditions by modern, energy-efficient lighting technologies, which can also help to keep the employees more concentrated, vigilant and productive, increase workplace safety and protect the environment by their integration into control networks. Another aspect to consider is that legislative regulations are becoming increasingly stringent. Especially in the industrial sector, the energy-saving potential is very high, a good example is night shifts work, where due to daylight absence, the lighting system is extensively used. A wide range of energy-efficient lighting technologies exists. This paper aims to give an overview of the new trends and application possibilities of the energysaving lighting systems for industrial buildings and halls. The main ideas are illustrated through the experiences of implemented projects.
\end{abstract}

Keywords: Illumination; sustainable; energy; industry.

\section{Introduction}

Sustainable lighting is an important business issue, not only to minimize environmental impact but also to comply with legislation, save energy costs and reduce the carbon footprint without affecting performance. Lighting is the way to a green future. Sustainability, which today meets our needs without compromising on the resources that future generations will need, affects everything we do as a society. Using only as much light as is needed when it is needed is key to reducing energy consumption [5]. The European Council implemented an energy efficiency action plan to reduce energy consumption by $20 \%$ by 2020 in European Council countries [10]. Another aspect to be taken into account is the fact that legislative regulations are becoming increasingly stringent. 
Especially in the industrial sector, the potential for energy savings is very high, that is because the lighting system is intensively used mainly due to night shifts and daylight absence in the halls and buildings. There is a wide range of energy-efficient lighting technologies. Most lighting projects are based on proposals to ensure uniformity of light intensity in the horizontal comparison plane, to maintain the recommended ratio of lighting and brightness between the place of the visual task and its immediate surroundings. It must be taken into account that the surfaces and colors of the room in which the lighting system is installed are an important part of the lighting system, as they affect the lighting in terms of its uniformity and in terms of the use of light energy [1], [4], [6].

In order to achieve the energy efficiency of the lighting system in buildings, standard organizations, such as the European Committee for Standardization, have developed standards to guide and provide specifications and requirements for technical systems. Lighting standards usually provide recommended values for the overall average intensity of illumination, ratios of illuminance at the areas of visual tasks and at their surroundings, the colour rendering indices CRI (expressing the rate of discolouration), the values of the glare index and the values of the correlated colour temperature CCT (STN EN 12 464-1). There are several options of lighting solutions, but for optimal selection, it is first necessary to know the work tasks, i.e. the visual tasks to be performed in the work area and select the type of lighting accordingly. Nowadays, there is a sufficiently large selection of lighting fittings at the market, having conventional, as well as modern design and, are suitable for any premises [1], [2]. There is an increasingly wide offer of lighting fittings and of lighting solutions that are using light-emitting diodes (LEDs) as light sources, which is indicating the future direction of development of the lighting technology. The basic characteristics of the lighting devices using LEDs, such as miniaturization, long service life (50,000 hours), efficiency, reliability, security, adjustability, instant start, clearly defined colours, are inspiring architects and lighting designers to search for new application possibilities. The transition to LED lighting also changes the way lighting control systems are designed [9]. LEDs are almost monochromatic light sources (half the bandwidth 20-30 nm) and provide a roughly linear light output in response to stimulation of the control signal.

\section{Materials and Methodology}

Based on strategies for the efficient use of lighting systems, an analysis of the effective use of lighting systems was performed and their synthesis with the possibilities of intelligent control, analyzes of energy-saving lighting systems was performed for the design and calculation of lighting systems. The results of rationalization procedures and measures adopted in order to reduce the energy intensity of lighting systems in industrial plants were the basis for the development of a methodological procedure for designing energy-efficient lighting systems in the field of mechanical engineering. The methodology presented in this paper is developed on the basis of applicable legislation for the assessment and evaluation of lighting systems in the work environment with regard to ensuring visual comfort and creating the required conditions for the visual activity, taking into account its energy efficiency and efficiency. The main parts of this methodology are divided into individual branches, the compilation of which creates a sequence of steps and conclusions for which the methodology was developed. This methodology is especially applicable to industrial halls. The basic steps can be described as follows:

- Assessment of the current state of the illuminated space/workshops.

- Evaluation of the working space according to the visual conditions of workers and comparison of parameters with technical - illuminated standards.

- Selection of lighting system components for several project variants.

- Calculations of lighting system variants.

- Selection of the optimal variant.

\subsection{Definition of the characteristics and calculation of the parameters}

When defining the characteristics of the technical equipment of the system, it is necessary to choose a suitable tool for calculating the lighting system for further progress in sustainable development. The calculation can be performed by various calculation methods (point-by-point method, lumen or light flux method etc.) or by computer tools intended for the elaboration of design and calculation of lighting systems. In this step, it is important to choose the appropriate type of calculation and take into account all the circumstances related to the lighting system.

A very important parameter that needs to be taken into consideration is the value of the maintenance factor, which is directly related to the design of the maintenance schedule for the lighting system. Determining the correct value of the maintenance factor has a great impact on the overall economy of the lighting system during its period of use.

Maintenance factors address the fact that lighting fixtures gradually reduce the amount of light they provide over several years, due to the deterioration of the lumen, dirt, faults, etc. The lighting scheme should be designed with the overall maintenance factor calculated for the selected lighting equipment, environment and specified maintenance plan. Every project is different, so the maintenance factor should be changed to suit the circumstances and the lighting technology used. The formula for the total maintenance factor (MF) for interior lighting is:

$M F=(L L M F x L S F) x L M F \times R M F$ 
Where: LLMF - lamp lumen maintenance factor, LSF - lamp survival factor, LMF - luminaire maintenance factor, RMF - room maintenance factor and SMF - surface maintenance factor. The result of the application of correct calculations and designs at this stage of design is a sophisticated optional solution that meets the requirements for health and safety at work and at the same time is energy efficient and economically advantageous.

\subsection{Lighting control system}

In the next stage of the lighting systems designing process, the elements of lighting control systems (Fig. 1) are implemented into the optional proposals, which offer considerable energy sources savings based on the implementation of sensors and daylight contribution, human motion sensors, time regimes introduced or by a creation of the luminaire groups and therefore it is important, considering the given type of the workplace, to assess all possibilities of the lighting control and regulation in the installation [5].

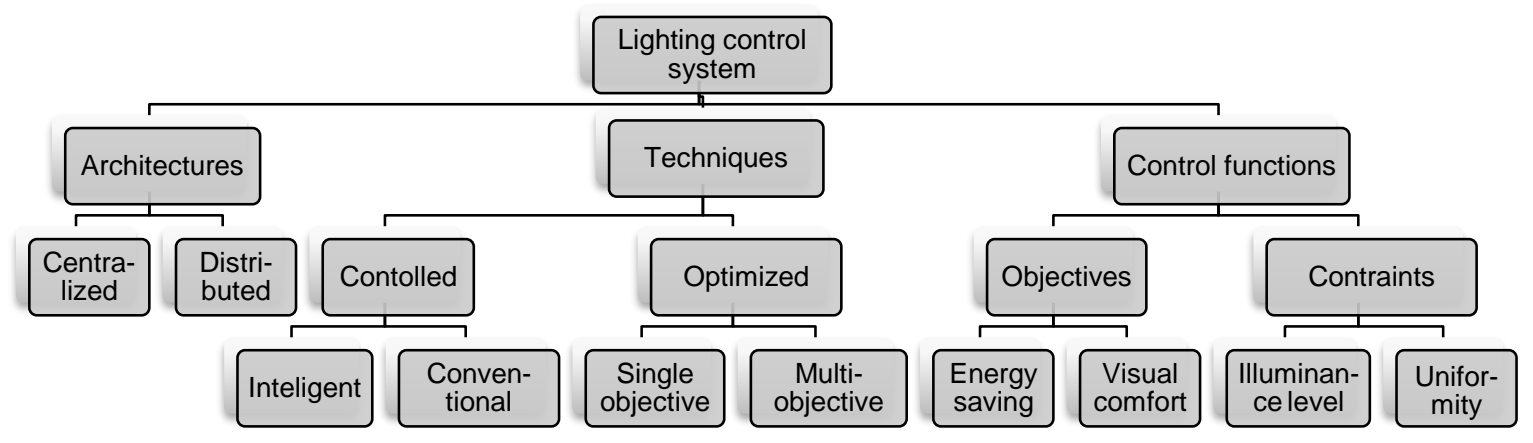

Fig. 1. Lighting control system scheme

The lighting control system can be divided into three main techniques: controlled, optimized and hybrid. Automatic lighting control systems reduce energy consumption by reducing the operating time of light bulbs based on various factors, such as occupancy, time of day, availability of daylight. There are various technologies that perform lighting control. Fig. 2 illustrates a general scheme for energy-efficient lighting control system [7].

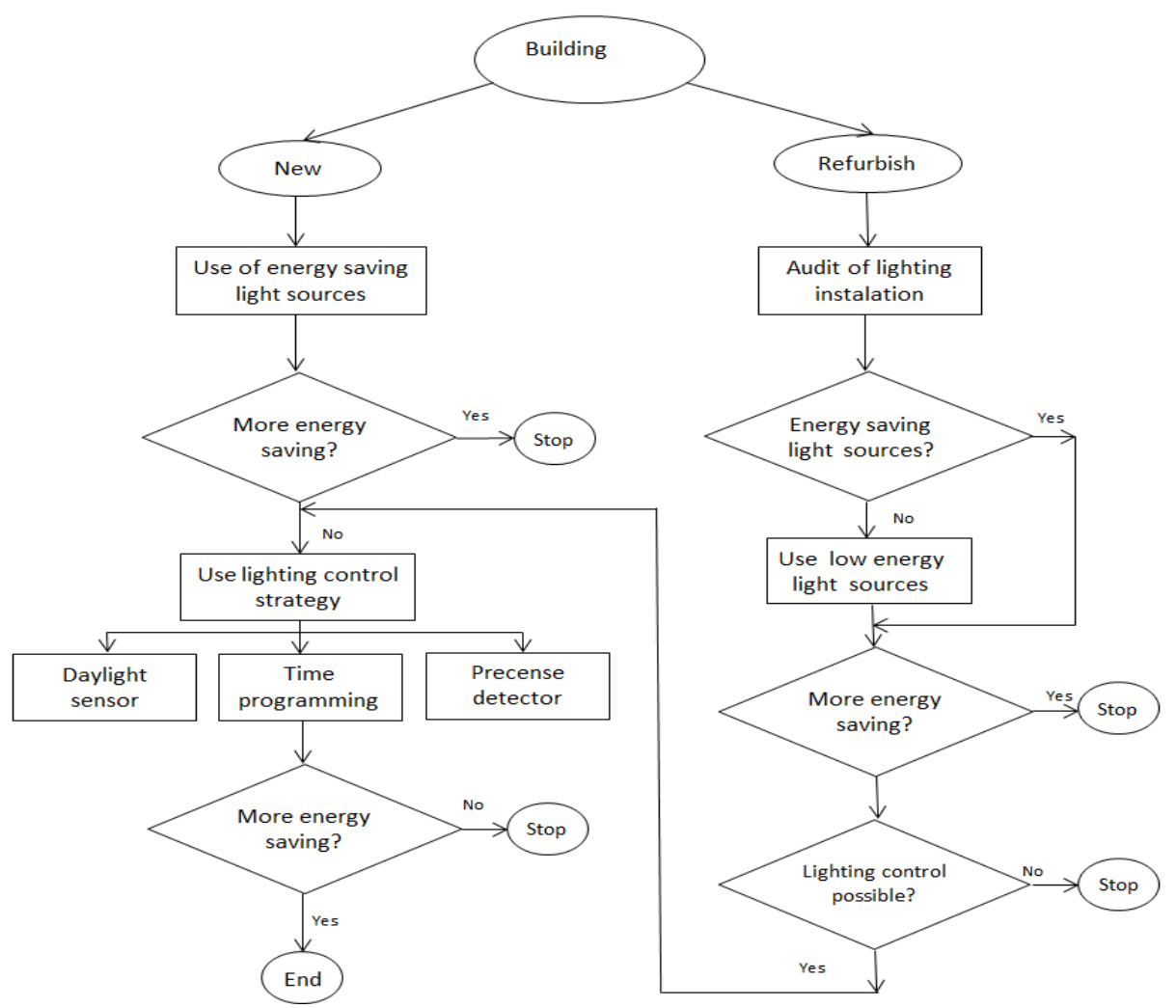

Fig. 2. General scheme for energy efficient design of lighting control 


\subsection{Digital addressable lighting interface}

Lighting control using DALI (Digital Addressable Lighting Interface) is a communication protocol for building lighting applications and is used to communicate between lighting control devices such as electronic ballasts, brightness sensors or motion detectors, providing energy-efficient solutions (Fig. 3). Lighting control systems use various communication protocols, such as DMX (Digital Multiplex Signal), RDM (Remote Device Management), KNX (Konnex Networks) and Digital Addressable Lighting Interface (DALI), to connect control devices to controllers and lighting fixtures. DALI is a widely accepted protocol and it is a standard communication protocol for lighting control. The interface and protocol are defined by the International Electrotechnical Commission as IEC 60929 / EN 60929 and have been modified by IEC 62386. DALI-2 indicates the latest version of the DALI protocol. For DALI-2, the IEC 62386 standard was revised at the end of 2014 to include many improvements and other new commands and functions [8].

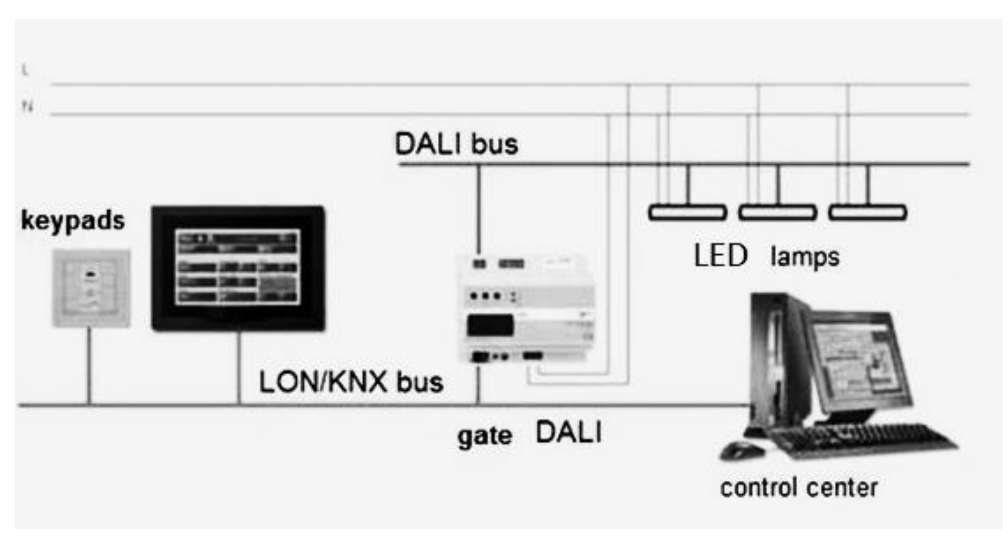

Fig. 3. Principle diagram of control system DALI

Most current digital ballasts and other electronic lighting devices (power switches, sensors and detectors, transformers, etc.) implement the DALI protocol. This is due to its simplicity in electrical installation and communication needs, which is basically based on two wires for the transmission of control signals for commissioning or other maintenance purposes. A typical DALI lighting control architecture includes a microcontroller that serves as a master controller for sending lighting control instructions and receiving status information to/from a DALI ballast that drives a bulb/LED lamp [9]. Lighting control and monitoring systems can be easily integrated with other technical equipment (e.g. HVAC), and so reduce energy consumption and improve overall comfort. Such solutions can enable an industrial workshop operator to provide the right amount of light where it is needed. The complexity of the lighting system with blinds, air conditioning and others is becoming essential for good integration and use not only in industry but also in various residential interiors. In the final stage of greening the lighting systems, the individual options are compared in terms of energy efficiency, total investment and operating costs. Lighting control strategy limits the operation time of the lighting system based on the occupancy time of space and actual daylight [3]. It enables a scheduling management strategy to reduce the operating hours of a lighting installation with automatic switching on or off based on schedules and occupancy patterns for different zones. Lighting control systems allow building operators to integrate lighting systems with other building services, such as heating, cooling, ventilation, in order to achieve a global energy approach for the whole building, in particular for green or energy-producing buildings. In the case of the reconstruction of the lighting system, a comparison of the original state and the proposed variant will be made, taking into account the energy savings and related financial savings, as well as the achieved lighting parameters of the system.

\section{Discussion}

The main goal of this contribution is to show effective lighting strategies based on a literature review. Future research will be focused on:

- Lighting system control solution, that fully complies with international standards for indoor lighting, e.g. standard EN12464-1.

- Application of open-source, low-cost and low-energy lighting control architecture.

- Easily reprogrammed and reconfigured lighting for integration with other automated building systems, capable of connecting wirelessly for control and monitoring purposes using one of the standard connection protocols (e.g. WiFi, Bluetooth).

- Dynamic loadable software control modules that provide DALI driver flexibility and save RAM storage, which is usually limited in embedded devices.

- Use of control systems for a variety of purposes, from personal lighting control needs and experimental research on the control of electronic ballasts and other control devices, instruments and sensors to advanced requirements in professional buildings, including energy management, maintenance and use of lighting. 


\section{Conclusion}

At present, during the 4th Industrial Revolution, it is important to pay increased attention to the design of lighting systems, not only in terms of energy efficiency aspects that will ultimately bring consumers financial savings, but also with regard to ensuring a safe working environment for employees. Energy efficient lighting solutions in industry can help reduce environmental impact and save costs while increasing production quality and employee productivity.

The exponential increase in the use of digital technologies can be observed in almost all areas of production. The concept of mass digitization of industrial production is gradually being implemented in the field of digital ergonomics. This can also be applied in the assessment of lighting as one of the important factors of the working environment. Further research will be focused on a comprehensive research of illumination factors (daylight, artificial, combined) of industrial halls using the tools of digital technologies as 3D digitization, visualization and simulation. The aim of the future research will be to create a digital twin of the illuminated space using elements of digital ergonomics. The efficient lighting solutions for the industry can help to reduce environmental impact and save on costs, at the same time as increasing quality, well-being and productivity.

The article should contribute to solving the problem of energy saving as one of the main goals of sustainable development. The aim of this article was to emphasize the importance of energy resources, to show the methodology of energy saving and efficient lighting applicable especially in industry, which can reduce the impact of $\mathrm{CO} 2$ emissions on the environment and save energy costs as well as overall costs. With the current climate crisis, the design of low-energy buildings must also take climate change into account.

\section{Acknowledgments}

This paper was written in the framework of the project No. KEGA 1/045/2018 „Transfer of the latest findings of research into processing textbook Environmental aspects of design engineering objects -Eco-design "solved at Technical University of Kosice and partly supported by the Student grant competition of the Technical University of Liberec under the project "Optimization of manufacturing systems, 3D technologies and automation" No. SGS-20195011.

\section{References}

[1] Despotović \& Zrakić, M. et al. (2014): Software environment for learning continuous system simulation In: Acta Polytechnica Hungarica Vol. 11, No. 2, 2014, pp. 187-202.

[2] Palcic, I. \& Buchmeister, B. (2016). Energy Efficiency in Slovenian Manufacturing Industry, Chapter 02. DAAAM International Scientific Book 2016, pp.011-024, B. Katalinic (Ed.), Published by DAAAM International, ISBN 978-3-902734-09-9, ISSN 1726-9687, Vienna, Austria.

[3] Dubois C. et al.(2015) Retrofitting the electric lighting and daylighting systems to reduce energy use in buildings: A literature review, Energy Res. J., vol. 6, pp. 25-41, 2015.

[4] Micieta, V. et al. (2015): Sustainable concept for green logistics and energy efficiency in manufacturing In: DAAAM International Scientific Book 2015. Chapter 33 pp. 391-400.

[5] Petek, J. - Glavic, P. \& Kostevsek, A. (2016): Comprehensive approach to increase energy efficiency based on versatile industrial practices In: Journal of Cleaner Production Vol. 112, 2016, pp. 2813-2821.

[6] Sözer, H. \& Takmaz, D. (2020 )Calculation of the Sensitivity factors within the Defined Indexes in a Building Level, Journal of Sustainable Development of Energy, Water and Environment Systems Vol. 8, Issue 1, pp 1-21, DOI: https://doi.org/10.13044/j.sdewes.d7.0265. Accessed on: 2020-08-13.

[7] Stuja, K.; Poszvek, G.; Wolfel, W. \& Markl, E.(2018): Integrated Method for the Design and Evaluation of Safety \& Secure Manufacturing Systems, Published by DAAAM International, ISBN 978- 3-902734-20-4, ISSN 17269679, Vienna, Austria DOI: 10.2507/29th.daaam.proceedings.022. Accessed on: 2020-03-11.

[8] Patel, M.; Mukherjee, S. (2017) Lighting Control Protocols and Standards. In Handbook of Advanced Lighting Techno-logy; Karlicek, R., Sun, C.-C., Eds.; Springer International Publishing AG: Cham, Switzerland, 2017; pp. $559-582$.

[9] George K. Adam: DALI LED Driver Control System for Lighting Operations Based on Raspberry Pi and Kernel Modules, Electronics 2019, 8(9), 1021; Available from: https://doi.org/10.3390/electronics8091021. Accessed on: 2020-08-13.

[10] The European Parliament and of the Council directive 2010/31/EU. Available from: https://www.eea.europa.eu/policy-documents/energy-performance-of-buildings-directive. Accessed on: 2020-0813. 